\title{
Optimising trial outcomes and patient retention for the MACRO trial for chronic rhinosinusitis*
}

\author{
Ngan Hong Ta' ${ }^{1}$ Claire Hopkins², Jane Vennik³ ${ }^{3}$ Carl Philpott1,4 \\ Norwich Medical School, University of East Anglia, Norwich, UK \\ 2ENT Department, Guy's and St Thomas' NHS Foundation Trust, London, UK \\ ${ }^{3}$ University of Southampton, Southampton, UK \\ ${ }^{4}$ ENT Department, James Paget University Hospital NHS Foundation Trust, Great Yarmouth, UK
}

Rhinology 57: 0, 000 - 000, 2019 https://doi.org/10.4193/Rhin19.142

*Received for publication:

April 5, 2019

Accepted: June 17, 2019

\begin{abstract}
Background: This study aimed to evaluate current subjective and objective outcome assessments for the MACRO (defining best Management for Adults with Chronic RhinOsinusitis) Trial which compares antibiotics, placebo and sinus surgery. This was to identify any redundant assessments and to include patient perspectives to determine acceptability for confirmation in the trial.
\end{abstract}

Methods : Adults CRS patients meeting the provisional eligibility criteria for the MACRO trial were recruited to this mixed-method study at 2 sites. Correlations between the objective outcome measures and SNOT-22 scores were evaluated. Selected participants took part in a semi-structured telephone interview to explore their experiences and views of undergoing outcome measures.

Results: Seventy patients (37\% male) were recruited, 36 had CRS without nasal polyps, 34 had CRS with nasal polyps. There was a weak inverse correlation between the SNOT-22 "Blockage" ratings and Peak Nasal Inspiratory Flow readings, a moderate inverse correlation between the SNOT-22 "Smell" ratings and Sniffin' Sticks scores, but no significant correlation between the SNOT-22 and Saccharin test results. The participants' experience of the trial visit was positive with an acceptable duration of trial visit. Most proposed outcome measures were valued by participants with the exception of the Saccharin test.

Discussion: The Sniffin'Sticks test and PNIF correlate with their respective component SNOT-22 scores but are considered important by patients; PNIF is simple, cheap test to perform. The Saccharin test will be removed as participants did not value it and was not highly rated in parallel work on a core outcome set for CRS.

Key words: outcome assessment, chronic rhinosinusitis, endoscopic sinus surgery, randomised controlled trial, patient selectionreported outcome measures

\section{Introduction}

Chronic Rhinosinusitis (CRS) is a common sinonasal syndrome but current guidance on treatments is limited by a paucity or low quality of evidence on which it is based. A series of Cochrane reviews evaluating the effectiveness of medical treatments in CRS confirmed the lack of high quality RCTs but also the lack of consistent outcome measures for this condition ${ }^{(1-3)}$. The European Position Paper on Rhinosinusitis and Nasal Polyps (EPOS 2012) ${ }^{(4)}$ emphasised that one of the research needs is to address this gap in the evidence base. In 2016, the UK National Institute of Health Research (NIHR) funded the MACRO Programme (De- fining best Management of Adults with Chronic RhinOsinusitis) for $£ 3.2$ million to include a 3-arm multi-centre randomised controlled trial (RCT) to compare longer-term antibiotics, placebo and endoscopic sinus surgery simultaneously ${ }^{(5)}$.

The selection of appropriate outcome measures is crucial in designing any multicentre RCTs. The chosen outcome measures should satisfy both requirements of being able to measure the effect of different interventions and to measure outcomes considered to be important to patients ${ }^{\left({ }^{6}\right)}$. With the proposed MACRO trial in mind, the research team were keen to ensure that any objective outcome measures provided meaningful information 
about how CRS patients respond to treatment and that they are acceptable to the participants. Furthermore, careful selection of outcome measures may help to increase patient recruitment and retention by reducing respondent burden and therefore engaging participants with follow-up trial visits.

In preparation for the MACRO trial, this study set out to determine the optimum set of outcome measures to use in the trial. The specific objectives were:

- To evaluate current subjective and objective outcome assessments for nasal syndromes

- To identify any assessments that are redundant for use in future trials

- To work with patient representatives and include their perspectives on the outcomes work undertaken.

\section{Materials and methods}

\section{Ethical approval}

The study was granted a favourable ethical opinion by the Essex Research Ethics Committee (REC reference number 17/EE/0020) and was sponsored by the University of East Anglia.

\section{Study design and settings}

A concurrent mixed methods study combining qualitative and quantitative methods was undertaken prospectively. Patients diagnosed with CRS attending outpatient clinics at the two proposed lead sites in the MACRO trial (James Paget University Hospital (JPUH) and Guys \& St Thomas' Hospital (Guys) and who met the eligibility criteria were invited to participate. All participants were taken through a simulated 'trial visit' (see below) and a selected group were then invited to take part in a semistructured telephone interview. The two stages of the study are demonstrated in Figure 1. The study recruited participants between March and November 2017. This study was performed in parallel to a project developing a core outcomes set (COS) for $\mathrm{CRS}$, the results of which were considered when informing final choice of trial outcomes.

Participants

CRS patients attending outpatient clinics at both recruiting sites were invited to participate. Patients were considered eligible for inclusion in the study if the following criteria were met: Inclusion criteria:

- Aged $\geq 18$

- Diagnosis of Chronic rhinosinusitis with (CRSwNP) or without polyps (CRSsNP) as per the EPOS 2012 guidelines ${ }^{(7)}$.

Exclusion criteria:

- Within 6 months post-operative

- Rare or complex sinus conditions

- CRS secondary to systemic disease (e.g. cystic fibrosis) or neoplasia
- Allergic fungal Rhinosinusitis (AFRS)

- Aspirin exacerbated respiratory disease

- Severe asthma (high doses of inhaled steroids i.e. $>1.5 \mathrm{mg}$ per day)

- Pregnant/lactating women

- Immunodeficiency states e.g. HIV

- Inability to give consent or to understand and comply with study instructions

\section{Variables}

The primary outcome measure chosen for the MACRO trial is the disease specific health related quality of life (HRQOL) SNOT-22 ${ }^{(8)}$ questionnaire. In this study, SNOT-22 was collected alongside generic HRQoL questionnaires: SF-12 ${ }^{(9)}$ and EQ-5D-5L (10). The asthma control test (ACT) was also conducted where applicable (11). Proposed objectives outcome measures for the MACRO trial that were evaluated included:

- Peak Nasal Inspiratory Flow (PNIF) to assess nasal airflow ${ }^{(12)}$

- Sniffin'Sticks test to assess olfactory function ${ }^{(13)}$

- Saccharin test to assess nasal mucocillary clearance (MCC) time ${ }^{(14)}$

- Peak expiratory flow rate (PEF)

All patients completed the SNOT-22 and the SF-12 at both sites; the EQ-5D-5L at JPUH only and the Asthma Control Test for selected cases. Only 10 patients were invited to undergo all of the objective assessments ( 5 at each site) to assess the complete MACRO 'trial visit' and to participate in interviews; the remainder of the participants underwent selected objective assessments according to the site to reduce participant burden:

- JPUH : Sniffin' Sticks test and Saccharin test

- Guys: PNIF, PEF and Asthma Control Test

\section{The nested qualitative interview process}

Semi-structured telephone interviews were conducted with as above. The interview guide, developed through discussions with the MACRO qualitative research team (Appendix 1), was used to structure the interviews. Each participant was asked the same questions outlined in the interview guide, but the guide remained sufficiently flexible to explore unforeseen topics.

Patients were asked to describe their overall experience of the simulated 'trial visit' and share their thoughts on the proposed objective outcome measures. They were also asked to suggest ways to encourage patient recruitment and retention in the future MACRO trial.

Data sources/measurement

1. PNIF: Three measurements were recorded and the best was taken as the final result ${ }^{(15)}$.

2. Sniffin' Sticks test: the extended test was performed to assess odour threshold (T), discrimination (D) and identification (I) giving a total TDI score out of $48^{(13)}$.

3. Saccharin test: A small sweetener tablet was placed under 


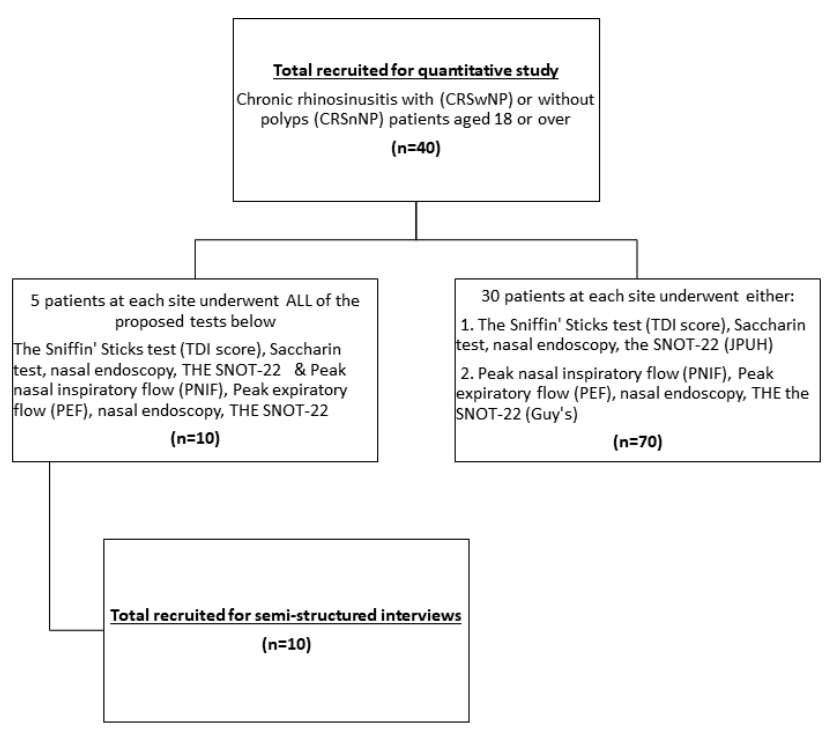

Figure 1. A STROBE flowchart illustrating participant involvement in 2 stages of the study.

the anterior end of the inferior turbinate on one side of the nose ${ }^{(16)}$. Time in minutes and seconds was recorded from placement of the tablet until the point at which participants could detect a sweet taste in the mouth.

4. Peak Expiratory Flow (PEF): PEF was included as an objective assessment of lower airways due to the prevalence of asthma in CRS ${ }^{(17)}$.

\section{Sample size}

A sample of 70 patients ( $95 \% \mathrm{Cl}$ width of 0.35 or less) is sufficient to detect a small sized $(r>0.3)$ association between the relevant SNOT-22 and the corresponding objective outcome measures with a statistical power of $80 \%$ and a $5 \%$ significant level.

\section{Statistical methods}

IBM SPSS Statistics $222^{(18)}$ was used to analyse the data. Pearson's correlation coefficients were calculated between each objective measures and what was thought to be the best fit item from the SNOT-22 as follows: smell and TDI scores, blockage and PNIF, rhinorrhoea (runny), Post Nasal Drip (PND), Thick Nasal Discharge (TND) and MCC. Further analysis with a simple linear regression to model the relationship between the pairs of variables was undertaken. The values of R2, adjusted R2 and F values of linear regression analyses were utilised to quantify the strength of the relationship between the exploratory variable (SNOT-22 smell, blockage, runny, TND, PND) and the "objective" variables (TDI score, PNIF and MCC time). Simple linear regression model assumptions were satisfied to be used in all variables after an assessment of scatter plots of residuals versus fitted values and normal quantile-quantile plots of the residuals.

\section{Qualitative analysis}

All interviews were audio-recorded, transcribed verbatim and checked against recording for accuracy by one researcher. NVivo 11, a qualitative research data management software, was used to facilitate inductive thematic analysis of data ${ }^{(19)}$. The analysis of the transcripts commenced with familiarisation and immersion in the data. Descriptive labels were used to systematically code the data. All frequently emerging codes were merged and grouped together in order to generate new sets of themes.

Table 1. Summary of objectives tool readings and PROMs ratings at the 'trial visit'.

\begin{tabular}{|c|c|c|}
\hline & Mean score (SD) & 'Healthy' values \\
\hline \multicolumn{3}{|l|}{ PROMs } \\
\hline Overall SNOT-22 ( $\mathrm{n}=70)$ & $42.3239(25.28567)$ & $\begin{array}{l}\text { A median score of 7-10 for males and 9-13 for females appears } \\
\text { 'normal' SNOT-22 }\end{array}$ \\
\hline SNOT-Blockage $(n=70)$ & $3.0704(1.60645)$ & \\
\hline SNOT-Smell $(n=70)$ & $3.1831(1.75100)$ & \\
\hline SNOT-Runny ( $n=70$ ) & $2.1972(1.60908)$ & \\
\hline SNOT-PND $(n=70)$ & $2.2113(1.86636)$ & \\
\hline SNOT-TND $(n=70)$ & $2.0141(1.76872)$ & \\
\hline SF-12 $(n=70)$ & 31.291667 (3.19964) & There is no 'healthy values'for SF-12, EQ-5D \\
\hline$E Q-5 D(n=35)$ & $76.0286(18.40953)$ & \\
\hline Asthma Control Test (ACT) $(n=22)$ & $18.6591(4.98900)$ & An ACT score $>19$ indicates well-controlled asthma \\
\hline \multicolumn{3}{|l|}{ Objective Outcome Measures } \\
\hline PNIF (litre/minute) $(n=40)$ & $15.2832(7.24020)$ & PNIF 'healthy values' vary depending on height, age and sex \\
\hline The Sniffin' sticks test (TDI score) $(n=55)$ & $18.1082(10.94979)$ & 31 and above \\
\hline Saccharin test (minutes) $(n=37)$ & $15.2832(7.24020)$ & Less than $10-20$ minutes \\
\hline Peak expiratory flow rate (PEF) (litre/minute) $(n=33)$ & $404.3333(137.16862)$ & PEF 'healthy values' vary depending on height, age and sex \\
\hline
\end{tabular}

$\mathrm{SD}=$ Standard Deviation, SNOT-22 $=22$ question Sino-Nasal Outcome Test 


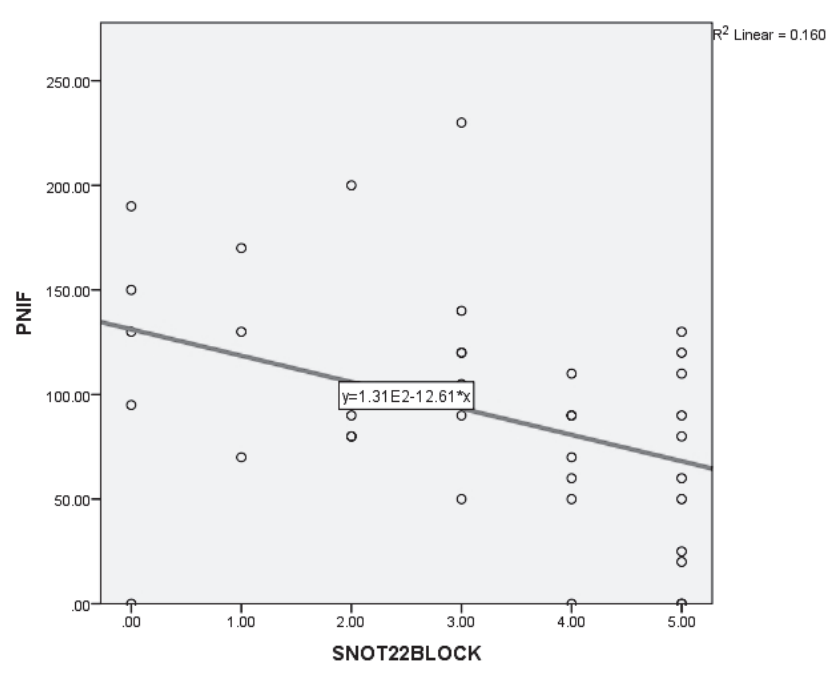

Figure 2. A scatter plot of PNIF readings and the SNOT-22 blockage ratings.

Inductive thematic analysis and telephone interviews were conducted simultaneously until thematic saturation of data was achieved.

\section{Results}

\section{Participants}

A total of 72 patients were screened for eligibility and 2 patients were excluded as they did not meet the criteria due to previous sinus surgery in the last 6 months or were found not to have CRS after CT scanning. The target of 10 patients to undergo all outcome measures and interviews for the qualitative component was achieved.

\section{Descriptive data}

The mean age of the included 70 patients was 53.3 years (SD of 16.77). A total of $50 \%$ included patients were male and $67 \%$ were diagnosed with CRSwNPs. Participant characteristics in the quantitative 'trial visit' are demonstrated in Table 2. Of the 10 patients who participated in a telephone interview, the mean age was 50 years, 3 participants were male and all were Caucasian.

\section{Outcome data}

All participants completed the SNOT-22 and SF-12 $(n=70)$; the EQ-5D was completed by JPUH participants $(n=35)$. Objective outcome measures were completed as follows: Sniffin' Sticks test 55 results, PNIF readings 40, Saccharine test 37 and PEF readings 33. The ACT was completed by 22 participants. A summary of all objective measurements and PROM ratings is outlined in Table 1. Mean and standard deviation (SD) were reported for all normally distributed variables.

\section{Main results}

Tables 3 and 4 illustrate the results of Pearson's correlation

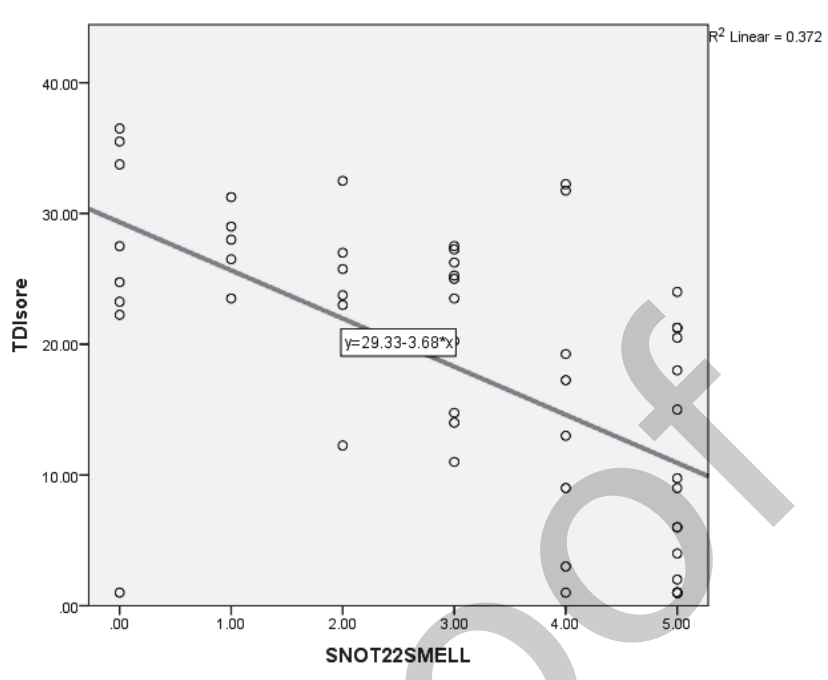

Figure 3. A scatter plot of TDI scores and the SNOT-22 smell ratings.

and simple linear regression analyses of the pairs of outcome measures.

\section{PNIF and "blockage"}

PNIF readings were found to be weakly correlated with the results of the SNOT-22 ratings for nasal blockage $(r=-0.400, p$ $<0.001)$. The spread of data is illustrated on the scatter plot (Figure 2).

\section{TDI score and "smell"}

A moderate inverse correlation was found between the Sniffin' Sticks test and the SNOT-22 ratings of smell $(r=-0.610, p<0.001)$. However, there was large variability of observed data and a high number of outliners shown on the scatter plot of the association (Figure 3). Further analysis with simple linear regression to examine the extent of the interaction of actual variance between the observed data, confirmed a weak correlation between the outcome measures with R2 of 0.372 .

\section{Other correlations}

The remaining pairs did not show any significant correlations:

- Saccharin test results and the SNOT-22 runny, TND, PND ratings

- PEF and ACT scores

- SF-12 and SNOT-22

- EQ-5D and SNOT-22

\section{The qualitative findings}

The inductive thematic analysis identified 3 broad themes including patients' feedback on the proposed outcome measures, their overall experience of the MACRO 'trial visit' and patients' suggestions for improving recruitment and retention of future studies. The themes are illustrated by quotations with partici- 
Table 2. Participant characteristics in the quantitative 'trial visit'.

\begin{tabular}{|lc|}
\hline $\begin{array}{c}\text { CRS, Mean (SD) } \\
\text { (n=70) }\end{array}$ \\
\hline Age (years), mean (SD) & $53.32,16.77$ \\
\hline Male (\%) & 50 \\
\hline $\begin{array}{l}\text { Types of CRS } \\
\text { CRSwNP } \\
\text { CRSsNP }\end{array}$ & 36 \\
\hline
\end{tabular}

CRSwNP = Chronic rhinosinusitis with nasal polyps, CRSsNP = Chronic rhinosinusitis without nasal polyps.

Table 3. Summary of Pearson's correlation coefficients with levels of significance showing only 2 significant correlations.

\begin{tabular}{|lll|}
\hline & $\begin{array}{c}\text { Pearson's } \\
\text { correlation } \\
\text { coefficient } \\
\text { estimate }\end{array}$ & P value \\
\hline SNOT-22 blockage and PNIF rates & $-0.400^{*}$ & 0.011 \\
\hline SNOT-22 smell and TDI scores & $-0.610^{*}$ & 0.000 \\
\hline SNOT-22 runny rating and MCC time & -0.171 & 0.486 \\
\hline SNOT-22 PND and MCC time & 0.090 & 0.600 \\
\hline SNOT-22 TND and MCC time & 0.242 & 0.155 \\
PEF readings and ACT scores & -0.029 & 0.897 \\
\hline SF-12 scores and SNOT-22 & 0.038 & 0.750 \\
\hline EQ-5D scores and SNOT-22 & 0.025 & 0.887 \\
\hline
\end{tabular}

${ }^{*} \mathrm{p}<0.05$ as determined by Pearson's correlation

Table 4. Summary of simple linear regression analysis with levels of significance showing a strong relationship between the two pairs of outcome measures.

\begin{tabular}{lllll} 
& \multicolumn{1}{c}{$\begin{array}{c}\text { Linear } \\
\text { regression } \\
\text { R2 estimate }\end{array}$} & $\begin{array}{c}\text { Linear } \\
\text { regression } \\
\text { Adjusted } \\
\text { R2 estimate }\end{array}$ & F value & P value \\
\hline $\begin{array}{l}\text { SNOT-22 blockage } \\
\text { and PNIF rates }\end{array}$ & $0.016^{*}$ & $0.138^{*}$ & $7.214^{*}$ & 0.011 \\
\hline $\begin{array}{l}\text { SNOT-22 smell and } \\
\text { TDI scores }\end{array}$ & $0.372^{*}$ & $0.360^{*}$ & $31.409^{*}$ & 0.000 \\
\hline
\end{tabular}

${ }^{*} \mathrm{p}<0.05$ as determined by simple linear regression analysis.

pant details presented in parentheses.

\section{Overall feedback on the proposed outcome measures}

Overall, a recurrent view was that patients described the majority of the proposed tests as relevant in measuring their CRS symptoms. None of the tests were found to be uncomfortable, painful or difficult to carry out in the simulated trial visit.

a. Sniffin' Sticks: Most participants welcomed the opportunity of undertaking the Sniffin' Sticks test finding it enjoyable and interesting ("I was blindfolded. I'm intrigued to know is it that my brain knows what it sees but my nose isn't. I'm interested to know what is actually going on there", patient 3). Additionally, some participants suggested the test to be valuable in assessing their symptom improvement over time ("I came away from the smell test thinking, wow, maybe I can go home and smell train and try and stimulate my sense of smell and, hopefully, get it back in a few months", patient 4).

b. PNIF: A recurrent sub-theme that emerged from the interviews was that PNIF was the most understood and appreciated test. Most participants considered PNIF to be easy and simple to participate in during their 'trial visit' ("I remember breathing in and out through a device connected to a mask. It was very easy to do. They obviously tested my blocked nose", patient 4). Additionally, some participants with asthma found PNIF to be familiar due to their prior experience of measuring PEF ("It was very straightforward. I've actually done sort of tests like that for my asthma. I know sinusitis it affects my breathing through my nose and the test measures that", patient 6).

c. MCC: Unlike the other tests, most participants could not recall undertaking the Saccharin test. Of those who did remember the test, concerns were expressed about the practical difficulties that occurred whilst taking the test in clinics ("That didn't work out very well. Something she did put inside my nose, which is a little, I don't know if it was a tablet or what it was. That tablet didn't dissolve very well. It wasn't very successful", patient 7). Moreover, most participants experienced confusion about how Saccharin test results would represent the severity of their CRS symptoms.

d. SNOT-22: Overall, the participants demonstrated an appreciation that SNOT-22 was easy to understand and the length of the questionnaire was considered to be appropriate for the trial visit. More importantly, the majority of patients found the SNOT-22 to be comprehensive, covering the most important CRS symptoms and impact on QoL ("The questions really hit the nail on the head. They're not just about what your symptoms, it's how you actually feel emotionally. It's like your emotional well-being which really suffers when you've got chronic sinusitis", patient 6).

\section{Overall 'simulated trial visit' experience}

There was a consensus of a good overall 'trial visit' experience reported by participants. Most participants found the experience pleasant and easy. Most participants expressed their belief that the average duration of a typical MACRO 'trial visit' experience of less than 1.5 hours was acceptable, even for full-time working patients. A common view amongst the participants was that the research nurses were enthusiastic and helpful, especially in explaining the different steps of the tests involved in the 'trial visit' ("The team was very helpful. I was really pleased", patient 8). Therefore, many participants expressed a willingness to partici- 
pate in the MACRO trial and similar studies in the future ("I' $d$ be happy to do anything to help research like this again as I think that it would improve things in the future.", patient 9).

\section{Participants' perspectives on ways to increase recruitment and retention}

In order to motivate patients to participate in similar studies in the future, participants appreciate a clear explanation of what would happen prior to agreeing to take part ("I liked that she explained exactly what we were going to do", patient 4) Easy to understand written information was considered to be important in assisting patients to decide whether to participate in a study. Additionally, good communication and clear verbal information delivered by approachable research staff was highly valued and recommended as a way to encourage participation from patients, to increase patient satisfaction, and to keep patients engaged for future trial visits.

Time commitment to attend a hospital-based study visit was described to be a potential barrier to participation and engagement, especially for full-time working participants. Therefore, shorter trial visits could be helpful in encouraging future participation in the MACRO trial. Patients indicated that they would like to be informed by the research team about any possible interpretations of their test results and the eventual outcomes of the study "I'd appreciate it if they send me a copy once it was all done and completed", patient 9). Consequently, updating patients on the study results via a letter or an email could be considered to enhance patient recruitment into future studies.

\section{Discussion}

\section{Key results}

We found a weak negative correlation found between the SNOT-22 blockage ratings and PNIF readings, and between the SNOT-22 smell ratings and TDI scores. PNIF has previously been shown to correlate weakly with symptomatic scores in CRS patients (both CRSsNP and CRSwNP) ${ }^{(20-22)}$. A recent study showed a relatively similar inverse correlation between pre-operative PNIF and pre-operative SNOT-22 $(r=-0.33)$ to our finding in this study. Our study only used the ratings of one SNOT-22 item for nasal blockage to correlate with PNIF readings, likely explaining the slightly stronger correlation found. As the test was valued by patients, and quick and easy to perform it was retained as one of our secondary outcome measures.

Apart from one study in CRSwNP patients (22), TDI scores have been shown previously to correlate strongly with PROMs (SNOT22, Visual analogue scales (VAS) or Questionnaire of Olfactory Disorders) measuring olfactory dysfunction in all types of CRS (both types of CRS, CRSwNP or CRSsNP, AFRS) (22-25) ( $r>-0.07$ ). TDI scores also correlated strongly with the SNOT-22 in one study in cases where the Lund Mackay Score was more than 15 out of 24 (24). Patients do however appear to value having this sense evalu- ated fully, and therefore again this outcome was retained. The SNOT-22 runny ratings did not correlate with Saccharin test results. The Saccharin test was not considered important to patients and reported to be challenging to undertake. Our study is the first to quantify the correlation between MCC with the SNOT-22 ratings in CRS patients. Other studies evaluated the correlation between the global SNOT-22 scores and nasal MCC measurements with the use of rhinoscintigraphy ${ }^{(26,27)}$. One paper examined the correlation between Saccharin test results and the global SNOT-22 scores in healthy patients which showed no correlation ${ }^{(28)}$. Our finding is consistent with the two previously mentioned studies also showing no correlation between objective readings and patient-rated ratings of nasal MCC. The COS for CRS studies ${ }^{(29)}$ recommended a minimum set of outcome measures to be included in future trials to facilitate systematic review and enable meta-analysis. Whilst the SNOT-22, Lund-Kennedy scores and The Sinus Control Test were considered essential outcome measures in the COS, the Saccaharin test was not considered important and thus the trial team opted to remove it from the final list of outcome measures ${ }^{(30)}$. No studies to date have examined patients' perspectives on PNIF, the Sniffin' Sticks test, and Saccharin test. The OMIPP project surveyed 80 rhinosinusitis patients and found that objective outcome measures of CRS symptoms were only mentioned in $3 \%$ of responses suggesting that primary outcome measures in future trials should be patient-rated to reflect the disease burden on patients ${ }^{(31)}$; this was confirmed by the COS. From thematic analysis of patients participating in OMIPP, nasal obstruction was shown to be the most frequently suggested and most important symptom for CRS sufferers ${ }^{\left({ }^{31}\right)}$. Impaired sense of smell was also rated to be a core CRS symptom; again this was also highly rated in the $\operatorname{COS}{ }^{299}$. Patients scored the objective evaluation of these core symptoms moderately ${ }^{(29)}$. However, runny nose symptoms were not regarded as CRS core symptoms ${ }^{(29)}$. and assessment of MCC with Saccharin testing was not considered to be important by patients ${ }^{(29)}$.

\section{Limitations}

The data collection at one site included more Sniffin' Sticks data and less PEF than intended. Although the effect of the missing data was automatically adjusted for, it reduced the reliability of the reported correlations. This shortfall is a frequently encountered challenge in research ${ }^{(32)}$ and highlights the burden on the sites to collect all outcome measures and ensure the ones collected are appropriate to avoid incomplete data and enhance patient recruitment and retention. Other available objective outcome measures could have been examined in our study such as rhinomanometry and other smell tests to assess olfaction ${ }^{(33)}$. The outcome measures were selected due to the potential implications for the trial in terms of time, local resources and the cost implications in a multi-centre trial. We chose to correlate the 
saccharin test with 'runny nose', PND, TND score of SNOT-22 but acknowledge that abnormalities of mucociliary transport may result in other symptoms. We also appreciate that muccocillary clearance and mucus production might be independent factors. Qualitative interviews rely on participants' ability to accurately and honestly recall their experience. Thus, the main limitation of our qualitive work is the possibility of recall bias from participants.

\section{Interpretation}

During the course of the study, the core outcome set (COS) for CRS studies was published ${ }^{(29)}$ that has recommended outcomes including the SNOT-22, Lund-Kennedy scores and The Sinus Control Test. The COS is recommended as minimum set of outcome measures to be included in future trials to facilitate systematic review and enable meta-analysis. Whilst future trials in CRS are expected to report the COS, researchers are encouraged to conduct research to select the most suitable additional outcome measures to reduce patient and research burden. We considered the recommendations of the COS when interpreting the findings from this study. Outcome measures should not only be able to detect the difference between the effect of trial interventions but also reflect aspects of the disease felt to be important to participants in the trial ${ }^{(6)}$. Therefore, the key strength of this study is its combined quantitative and qualitative approach but also at the study sample is representative of the upcoming MACRO trial with a balanced number of both CRS.

\section{Generalisability}

Our literature search highlighted that there is limited literature on the correlation between objective outcome measures and PROMs in sinonasal syndromes, especially in CRS ${ }^{(20,22,26-28,34)}$. To our knowledge, our study is the first paper to examine the correlation between MCC measurements using Saccharin test and the SNOT-22 ratings in CRS patients. Overall, our quantitative study results have added to the currently limited literature on the topic.

\section{Conclusion}

This study is the first to assess suitability of proposed objective outcome measures for a CRS trial using a combined approach of considering both statistical analysis of outcome data and participants' perspectives. Whilst this study was designed specifically for the MACRO trial, its use of a mixed methods research model to select the most appropriate objective tools for a large $\mathrm{RCT}$, can be replicated elsewhere to reduce patient and research burden within the trial.

\section{Acknowledgements}

Abigail Walker, Allan Clark, Jane Wood, Teresa Ferreira, Abigail Tetteh.

An NIHR Programme Grant supported the salaries of the Chief Investigators in conducting this pre-trial study (funding ref: RPPG-0614-20011).

\section{Author contribution}

All authors have made substantial contributions to the article.

\section{Conflict of interest}

None declared.

\section{References}

1. Head K, Chong LY, Piromchai $P$, et al Systemic and topical antibiotics for chronic rhinosinusitis. The Cochrane database of systematic reviews. 2016:4:Cd011994.

2. Head K, Chong LY, Hopkins C, Philpott C, Schilder AG, Burton MJ. Short-course oral steroids as an adjunct therapy for chronic rhinosinusitis. The Cochrane database of systematic reviews. 2016;4:Cd011992.

3. Head K, Chong LY, Hopkins C, Philpott C, Burton MJ, Schilder AG. Short-course oral steroids alone for chronic rhinosinusitis. The Cochrane database of systematic reviews. 2016:4:Cd011991

4. Fokkens WJ, Lund VJ, Mullol J, et al. European Position Paper on Rhinosinusitis and Nasal Polyps 2012. Rhinol Suppl. 2012(23):3 p preceding table of contents, $1-298$.

5. Treat Yourself Better with Advice from a Pharmacist Campaign [Available from: http://www.treatyourselfbetter.co.uk

6. Williamson PR, Altman DG, Blazeby JM, et al. Developing core outcome sets for clinical trials: issues to consider. Trials. 2012;13(1):132.

7. Fokkens WJ, Lund VJ, Mullol J, et al. EPOS 2012: European position paper on rhinosinusitis and nasal polyps 2012. A summary for otorhinolaryngologists. Rhinology. 2012;50(1):1-12.

8. Hopkins C, Gillett S, Slack R, Lund VJ, Browne JP. Psychometric validity of the 22-item Sinonasal Outcome Test. Clin Otolaryngol. 2009;34(5):447-54.

9. Ware J, Jr., Kosinski M, Keller SD. A 12-Item Short-Form Health Survey: construction of scales and preliminary tests of reliability and validity. Med Care. 1996;34(3):220-33.

10. Herdman M, Gudex C, Lloyd A, et al. Development and preliminary testing of the new five-level version of EQ-5D (EQ-5D5L). Qual Life Res. 2011;20(10):1727-36.

11. Nathan RA, Sorkness CA, Kosinski M, et al. Development of the asthma control test: a survey for assessing asthma control. J Allergy Clin Immunol. 2004;113(1):59-65.

12. Holmstrom M. The use of objective measures in selecting patients for septal surgery.
Rhinology. 2010;48(4):387-93.

13. Hummel T, Sekinger B, Wolf SR, Pauli E, Kobal G. 'Sniffin' sticks': olfactory performance assessed by the combined testing of odor identification, odor discrimination and olfactory threshold. Chem Senses. 1997;22(1):39-52.

14. Andersen I, Camner P, Jensen PL, Philipson K, Proctor DF. Nasal clearance in monozygotic twins. Am Rev Respir Dis. 1974;110(3):301-5.

15. Chapter 1.2 - Investigation of nasal disease. In: Maran AGD, editor. Logan Turner's Diseases of the Nose, Throat and Ear (Tenth Edition): Butterworth-Heinemann; 1988. p. 13-20.

16. Rutland J, Cole PJ. Nasal mucociliary clearance and ciliary beat frequency in cystic fibrosis compared with sinusitis and bronchiectasis. Thorax. 1981;36(9):654-8.

17. Philpott CM, Erskine S, Hopkins C, Kumar N, Anari S, Kara N, et al. Prevalence of asthma, aspirin sensitivity and allergy in chronic rhinosinusitis: data from the UK National Chronic Rhinosinusitis Epidemiology Study. 
Respir Res. 2018;19(1):129.

18. $\mathrm{IBM}^{\oplus}$. IBM ${ }^{\circledast} \mathrm{SPSS}^{\circledast}$ Statistics V22.0

19. Braun V, Clarke V. Using thematic analysis in psychology. Qual Res Psychol. 2006;3(2):77101.

20. Whitcroft KL, Andrews PJ, Randhawa PS Peak nasal inspiratory flow correlates with quality of life in functional endoscopic sinus surgery. Clin Otolaryngol. 2017;42(6):118792.

21. Proimos EK, Kiagiadaki DE, Chimona TS, Seferlis FG, Maroudias NJ, Papadakis CE. Comparison of acoustic rhinometry and nasal inspiratory peak flow as objective tools for nasal obstruction assessment in patients with chronic rhinosinusitis. Rhinology. 2015;53(1):66-74.

22. Hox V, Bobic S, Callebaux I, Jorissen M, Hellings PW. Nasal obstruction and smell impairment in nasal polyp disease: correlation between objective and subjective parameters. Rhinology. 2010;48(4):426-32.

23. Simopoulos $E$, Katotomichelakis $M$ Gouveris H, Tripsianis G, Livaditis M Danielides V. Olfaction-associated quality of life in chronic rhinosinusitis: adaptation and validation of an olfaction-specific questionnaire. Laryngoscope. 2012;122(7):1450-4.

24. Chung JH, Lee YJ, Kang TW, et al. Altered Quality of Life and Psychological Health (SCL-90-R) in Patients With Chronic Rhinosinusitis With Nasal Polyps. Ann Otol Rhinol Laryngol. 2015;124(8):663-70.
25. Philpott CM, Thamboo A, Lai L, et al Olfactory dysfunction in allergic fungal rhinosinusitis. Arch Otolaryngol Head Neck Surg. 2011;137(7):694-7.

26. Athanasopoulos I, Naxakis S, Vlastos IM, et al. Is mucociliary transport velocity related to symptoms in chronic rhinosinusitis patients? Hell J Nucl Med. 2008;11(1):30-2.

27. Naxakis S, Athanasopoulos I, Vlastos IM, Giannakenas C, Vassilakos P, Goumas P. Evaluation of nasal mucociliary clearance after medical or surgical treatment of chronic rhinosinusitis. Eur Arch Otorhinolaryngol. 2009;266(9):1423-6.

28. Boatsman JE, Calhoun KH, Ryan MW Relationship between rhinosinusitis symptoms and mucociliary clearance time. Otolaryngol Head Neck Surg. 2006;134(3):491-3.

29. Hopkins C, Hettige R, Soni-Jaiswal A, et al. CHronic Rhinosinusitis Outcome MEasures (CHROME), developing a core outcome set for trials of interventions in chronic rhinosinusitis. Rhinology. 2018;56(1):22-32.

30. Philpott C, le Conte S, Beard D, et al. A Randomised Controlled Tria of Clarithromycin and Endoscopic Sinus Surgery for Adults with Chronic Rhinosinusitis with and without nasal polyps: study protocol for the MACRO randomised controlled trial. Trials. 2019;20(1):246

31. Hopkins C, Philpott C, Crowe S, et al.
Identifying the most important outcomes for systematic reviews of interventions for rhinosinusitis in adults: working with Patients, Public and Practitioners. Rhinology. 2016;54(1):20-6.

32. Kang $\mathrm{H}$. The prevention and handling of the missing data. Korean J Anesthesiol. 2013;64(5):402-6.

33. Hussain M, Maran AGD, Hussain SM Logan Turner's Diseases of the Ear, Nose and Throat, 11th Edition: Head and Neck Surgery, 11th Edition. London, UNITED KINGDOM: Chapman and Hall/CRC; 2015.

34. Chung JH, Lee YJ, Kang TW, et al. Altered Quality of Life and Psychological Health (SCL-90-R) in Patients With Chronic Rhinosinusitis With Nasal Polyps. Ann Otol Rhinol Laryngol. 2015;124(8):663-70.

\section{Ngan Hong Ta}

Norwich Medical Schoo

Faculty of Medicine and Health

University of East Anglia

Norwich NR4 7TJ

United Kingdom

E-mail:t.ngan@uea.ac.uk 
APPENDIX 1. The semi-structured interview guide 1.

\section{NHS \\ Health Research Guy's and St Thomas' W/HS NHS Foundation Trust \\ UE】 \\ James Paget University Hospitals WHS NHS Foundation Trust}

Outcome Assessment for Chronic Rhinosinusitis

\section{PATIENT INTERVIEW GUIDE}

Below is a list of topics/questions to be discussed in this study. This qualitative work will remain
flexible with respect to participants' agendas but we will cover the broad topics/questions noted.

AIMS AND OBJECTIVES:

The aim of this interview is to understand participants' views and experiences of having been

involved in the study. The interview will specifically explore:

1. Participants' views of the acceptability of the individual assessments used in the study

2. Participants' experience of participating in the study such as recruitment and study process

\section{INTRODUCTION:}

Introduce self and purpose of the interview:

- Remind them about the study they participated in and the signed consent form to be

interviewed today

he participant that the recorder is on. Check if they are happy with this.

Ask if they have any questions

Deconfirm written consent:

- Ask if the patient is still happy with the signed consent form and happy to proceed with the phone interview

Reassure the participate:

- All content discuss will be kept confidential

\section{National Institute for Health Research}

Guy's and St Thomas' WH'S NHS Foundation Trust

- No direct quotes will be used to identify them as an individual

- They can change their mind and can stop the interview at any time

- They can decline to answer any question

- The interview will take approximately 15 minutes.

$>$ Check if the participant has any question

Ask the participant to alert the interviewer if there is any problem with the telephone line at any point throughout the interview

Dnsure that the audio-recorder is on/working at this point

\section{QUALITATIVE QUESTIONS:}

\section{Section 1 - The overall experience of participating in the study}

You recently took part in our study looking at outcome assessments for patients with chronic sinusitis (CRS). In 2018 we will be starting a clinical trial for patients with CRS and we will be using some or all of these assessments to see how patients in the trial respond to the treatments. I would like to firstly start asking you some questions about participating:

- Firstly, please could you talk me through your experiences of taking part in the study

- Prompt: What specifically did you like/dislike about the study?

- Prompt: what did you feel about being asked to participate?

Prompt: How did you find the research staff, time taken, benefits, harms?

Section 2 - The overall feedback of the individual assessments in the study

- Please could you describe your overall experiences of the assessments/tests for CRS that you have undergone in our study

- Prompt: How do you feel about the information that was given to you about the what was involved in undergoing the test?

Prompt: How would you feel about undergoing this set of tests at 3 visits as part of a trial for CRS?

- Prompt: what do you think about the time it took to complete the assessment?

- Could you describe any potential benefits and harms you thought were associated with the assessment?

Prompt: views on the whole assessment visit?

- I would now like to ask you about the individual tests that you took as part of the assessment. Firstly, can I ask you about....

1. Objective tests:
NHS

National Institute for Health Research

\section{Guy's and St Thomas' WWS} NHS Foundation Trust

- A smell test (called Sniffin' Sticks)

- A saccharin test (to see how fast mucus flows through your nose)

- A peak expiratory flow test (breathing out through mouth into a device)

- A peak nasal inspiratory flow test (sniffing in through nose through a device)

- Rhinomanometry (airflow measurement with mask and small nostri bung)

2. Subjective questionnaires:

- Asthma control test (questions about asthma medication use) - if completed

- SF-12 (questions about your health in general)

- EQ-5D-5L (questions about your health in general)

- SNOT-22 (questions about your CRS)

Prompt: What specifically did you like/dislike about the assessment?

Section 3 - Recruitment and retention:

- Just for studies in general, how do you think we could encourage recruitment onto this kind of study in the future?

- Prompt: What would you recommend to improve the experience? (Recruitment)

Prompt: What do you think would help to keep people engaged in this sort of study? (Recruitment Retention)

Prompt: Is there anything that you would deter you from continuing in a trial that included these tests/questionnaires? (Retention)

Prompt: Can you see why people might drop out of these studies? (Retention)

What else you would like us to offer to you after you have completed the tests and the questionnaires?

What additional information would you like after you have completed the tests and the questionnaires? ( results of the tests may be a benefit we can offer them?)

TURN OFF THE RECORDER AND DEBRIEF:

D Ask if the participant has any questions about the study.

Revisit consent - are you still happy for the interview to be used (anonymously)?

Tell participant that the audio recorder is now switched off

$>$ Thank participant for taking part in the interview 